hep-th/0411268

NSF-KITP-04-128

\title{
How Hairy Can a Black Ring Be?
}

\author{
Gary T. Horowitz ${ }^{1}$ and Harvey S. Reall ${ }^{2}$ \\ ${ }^{1}$ Department of Physics, UCSB, Santa Barbara, CA 93106 \\ gary@physics.ucsb.edu \\ ${ }^{2}$ Kavli Institute for Theoretical Physics, UCSB, Santa Barbara, CA 93106 \\ reall@kitp.ucsb.edu
}

\begin{abstract}
It has been shown recently that there is a large class of supersymmetric solutions of five-dimensional supergravity which generalize the supersymmetric black ring solution of Elvang et al. This class involves arbitrary functions. We show that most of these solutions do not have smooth event horizons, so they do not provide examples of black objects with infinite amounts of "hair".
\end{abstract}




\section{Introduction}

In four dimensions, there is considerable evidence to support Wheeler's idea that black holes have no hair. The traditional statement that stationary black holes in Einstein-Maxwell theory are uniquely characterized by their mass, charges and angular momentum was established by the uniqueness theorem for the Kerr-Newman black hole. There are also results showing that gravity coupled to other simple matter fields (e.g. scalar fields with suitable potentials) does not yield any new stationary black hole solutions [1]. Over the past decade, it has been shown that more complicated matter fields can yield new black holes. In particular, many theories with gravitating solitons have new black hole solutions, since one can put a small black hole inside the soliton [2]. However, even in these cases, it remains true that the black holes are characterized by a small number of parameters. Most of the information that falls into the black hole is (classically) lost. The spirit of the "no-hair theorem" holds.

In more than four dimensions, much less is known. Even for pure gravity, stationary fivedimensional black holes are not uniquely characterized by their mass and angular momenta. There exist "black rings" - black holes of topology $S^{1} \times S^{2}$ - that have the same conserved quantities as black holes of spherical topology [3]. If electromagnetic fields are included, then the black rings can carry charge [4] and, moreover, the number of parameters required to specify black ring solutions now exceeds the number of conserved quantities that they carry [5]. This remains true even when one imposes the strong constraint of supersymmetry. A family of $U(1) \times U(1)$ invariant supersymmetric black rings has been found [6, 7, 8, 9] and these solutions are not characterized by their asymptotic charges. Nevertheless, for all of these examples, only a finite number of parameters are required to specify the solution so again the spirit of the no-hair theorem is retained.

Recently, a more serious challenge to the idea that black holes have no hair has arisen. Bena and Warner (BW) have claimed [7] that there exists a family of supersymmetric black ring solutions described by arbitrary functions!

BW showed how to construct a family of supersymmetric solutions of five-dimensional supergravity that is specified by seven arbitrary functions of one variable. Four of these functions describe the embedding of a curve $\mathcal{C}$ into $R^{4}$ and the other three specify certain charge densities along $\mathcal{C}$. If $\mathcal{C}$ is taken to be a round circle with constant charge densities then this construction reproduces the $U(1) \times U(1)$ invariant black ring solutions. BW claimed that these restrictions on the functions are unnecessary and that black ring solutions exist for

any smooth non-intersecting closed curve $\mathcal{C}$ with essentially arbitrary charge densities. They based this claim on the following argument. $\mathcal{C}$ can be approximated locally by a straight line with constant charge density. The solution in which $\mathcal{C}$ is exactly straight with constant 
charge density was constructed in [10] and describes an infinite black string with a smooth horizon. Hence the solution for arbitrary $\mathcal{C}$ with varying charge density should behave locally like that of [10] and therefore have a regular horizon.

We will show that this argument is incorrect: most of these solutions do not have smooth horizons and hence cannot be regarded as black ring solutions. In fact our arguments suggest that the only solutions which are smooth belong to the original $U(1) \times U(1)$ invariant family (or superpositions of them [11, 9]). The situation is similar to a straight extremal black string in five or six dimensions wrapped around an $S^{1}$. Such solutions have a null translational symmetry so one can add various types of traveling waves described by arbitrary functions 12. Soon after they were discovered, these waves were interpreted in terms of "classical hair" 13. However, it was later shown that there is a problem with this interpretation. After the waves are added, the metric is still continuous at the horizon and all scalar curvature invariants remain finite [14]. Nevertheless, it turns out that there is a curvature singularity at the horizon because certain curvature components in a basis parallelly propagated along a geodesic will diverge there [15, 16]. More physically, a freely falling observer will experience infinite tidal forces. This is a result of the fact that the waves travel around the circle an infinite number of times, accumulating near the horizon.

The problem with the BW solutions is similar. The BW argument assumes that a point on the curve $\mathcal{C}$ in $R^{4}$ corresponds to a point on a horizon in spacetime (more precisely, a point on the $S^{1}$ of a $S^{1} \times S^{2}$ horizon). However, we shall see that an actual horizon requires a limit $\ell \rightarrow \infty$ where $\ell$ is the parameter along the curve. ${ }^{1}$ So any non-trivial periodic function of $\ell$ (such as the charge densities on $\mathcal{C}$ ) will run through an infinite number of periods before crossing the horizon and hence will not be continuous there. We will use this to show that the metric does not admit a smooth horizon.

More precisely, we will show that the metric cannot be extended through a $C^{2}$ horizon. The similarity with the results of [15, 16] suggests that there will be a parallelly-propagated curvature singularity although we have not proved this. Although $C^{2}$ is the minimum $\operatorname{degree}$ of differentiability usually demanded of spacetime, one might ask whether the metric can be extended through a horizon of lower differentiability, such as the $C^{0}$ horizon of [14], as would be required to assign an area to the horizon. This remains an open question. ${ }^{2}$ In any case, the lack of a smooth horizon shows that these solutions do not really describe black holes with infinite hair of the type envisaged by Wheeler.

\footnotetext{
${ }^{1}$ This is because the solution rotates in the $\ell$ direction. This behaviour is familiar from the Kerr solution, for which the azimuthal angle of the Boyer-Lindquist coordinates diverges at the horizon.

${ }^{2}$ Note that the four dimensional uniqueness theorems assume smoothness (in fact analyticity) of the horizon so if one permits horizons of lower differentiability then it might be possible to violate black hole uniqueness even in four dimensions.
} 
The $U(1) \times U(1)$ invariant black rings evade our argument because the charge densities are constant and $\mathcal{C}$ follows the orbit of a Killing field of $R^{4}$ so nothing depends on $\ell$. Hence the limit $\ell \rightarrow \infty$ can be taken and a smooth extension through a horizon found, as described in [6, [8, 9].

In the next section we review the equations that govern supersymmetric solutions of fivedimensional supergravity and BW's method of solving them. We also clarify the (surprisingly subtle) relation between the charge density on the ring and the total charge of the spacetime. The difficulties of having a smooth horizon can be seen even in the simple case when the curve $\mathcal{C}$ is a straight line, so we discuss this first, in section 3 . The case of an arbitrary curve is investigated in section 4 . The final section contains some further discussion.

\section{Supersymmetric solutions of 5D supergravity}

If we set all three charge densities equal, then the solutions of $\mathrm{BW}$ become solutions to minimal five-dimensional supergravity. The bosonic sector of this theory is just EinsteinMaxwell theory with a Chern-Simons term. Any supersymmetric solution of this theory admits a globally defined non-spacelike Killing vector field $V$ [17]. In a region where this is timelike, any supersymmetric solution takes the form [18]:

$$
\begin{aligned}
d s^{2} & =-f^{2}(d t+\omega)^{2}+f^{-1} h_{m n} d x^{m} d x^{n} \\
F & =\frac{\sqrt{3}}{2} d[f(d t+\omega)]-\frac{1}{\sqrt{3}} G^{+}
\end{aligned}
$$

where $V=\partial / \partial t, h_{m n}$ is a hyper-Kähler metric on a four-dimensional "base space" $\mathcal{B}, f$ and $\omega$ are a scalar and 1 -form on $\mathcal{B}$, and $G^{+}$is defined by

$$
G^{+}=\frac{1}{2} f(d \omega+\star d \omega),
$$

where $\star$ denotes the Hodge dual with respect to $h_{m n}$ with orientation chosen so that the complex structures are anti-self-dual. The equations of motion for the theory reduce to the following equations on $\mathcal{B}[18$ ]

$$
\begin{aligned}
d G^{+} & =0, \\
\Delta f^{-1} & =\frac{4}{9}\left(G^{+}\right)^{2} .
\end{aligned}
$$

where $\left(G^{+}\right)^{2} \equiv \frac{1}{2}\left(G^{+}\right)_{m n}\left(G^{+}\right)^{m n}$ with indices raised with the base space metric $h_{m n}$ and $\Delta$ is the Laplacian on $\mathcal{B}$.

A good understanding of these equations was lacking until Bena and Warner made the important discovery [7] that they can be solved in a linear manner as follows. For simplicity, 
we take the base space to be flat: $\mathcal{B}=R^{4}-\mathcal{C}$ where $\mathcal{C}$ is a smooth curve without selfintersections. The first step is to solve (2.3). Introduce a current $J$ on $\mathcal{C}$ and let $G$ solve the Maxwell equations for this current on $R^{4}$, i.e., $d \star G=\star J, d G=0$. Now set $G^{+}=G+\star G$ on $R^{4}-\mathcal{C}$. This gives

$$
d G^{+}=\star J
$$

and hence (2.3) is satisfied on $R^{4}-\mathcal{C}$ because $J$ is supported on $\mathcal{C}$.

Having determined $G^{+}$, the next step is to solve (2.4) to determine $f$. BW present an expression for the general solution using a Green's function integral. However, this integral does not converge because $G^{+}$diverges as $1 / d^{2}$ where $d$ is the proper distance from $\mathcal{C}$, so the right hand side of (2.4) diverges as $1 / d^{4}$ and therefore is not integrable. Fortunately, this problem can be circumvented as follows.

Consider a Poisson equation

$$
\nabla^{2} \phi=s_{0}+s_{1}
$$

where $s_{0}$ and $s_{1}$ are smooth functions on $R^{4}-\mathcal{C}$ and $R^{4}$ respectively, and $s_{0}+s_{1}$ vanishes sufficiently rapidly at infinity. Let $\mathcal{T}$ denote a neighborhood of $\mathcal{C}$. Then the solution satisfying $\phi=\bar{\phi}$ at infinity is given by $\phi=\phi_{0}+\phi_{1}$ where $\phi_{0}$ obeys

$$
\nabla^{2} \phi_{0}=s_{0} \quad \text { in } \mathcal{T}
$$

and $\phi_{0}$ is chosen to decay smoothly to zero outside $\mathcal{T}$ (without satisfying any particular equation). $\phi_{1}$ is defined as the unique solution of

$$
\nabla^{2} \phi_{1}=s_{0}+s_{1}-\nabla^{2} \phi_{0}
$$

obeying $\phi_{1}=\bar{\phi}$ at infinity. The right hand side of this equation is smooth on $R^{4}$, so $\phi_{1}$ can be determined using a Green's function, and is smooth on $R^{4}$. Note that the singular part of $\phi$ is determined entirely by $\phi_{0}$. In other words, we can determine the singular part of $\phi$ just by solving the Poisson equation in the neighborhood of $\mathcal{C}$ without worrying about the boundary condition at infinity, as one would expect physically. Any two solutions of (2.6) that obey the same boundary condition at infinity differ at most by a harmonic function on $R^{4}-\mathcal{C}$ that vanishes at infinity. Such a function can be regarded as arising from a distributional source on $\mathcal{C}$.

Carrying out this procedure for equation (2.4) determines $f^{-1}$ up to the addition of a function harmonic on $R^{4}-\mathcal{C}$ which can be regarded as arising from a charge distribution $\rho$ on $\mathcal{C}$ so, using differential forms, we write

$$
d \star d f^{-1}+\frac{4}{9} G^{+} \wedge G^{+}=4 \pi \rho \delta[\mathcal{C}] \star 1,
$$


where $\rho$ is the charge density on $\mathcal{C}$ and $\delta[\mathcal{C}]$ is a delta-function supported on $\mathcal{C}$.

BW arrived at the surprising conclusion that the total charge of the spacetime is not determined entirely by $\rho$ but also has a contribution from fluxes. We shall see that this is incorrect. The confusion arises from subtleties in the definition of $\rho$ so it is worth discussing this at some length.

First, note that we have to move the singular $\left(G^{+}\right)^{2}$ term to the left hand side of (2.9) in order to interpret the RHS as a distribution. In practice, we will use this equation to determine $\rho$ once we have a solution for $f$ and $G^{+}$. The solution for $f$ will involve an arbitrary coefficient and (2.9) can be used to relate this coefficient to $\rho$. The obvious way to do this is to integrate over a tubular neighbourhood $\mathcal{T}$ of $\mathcal{C}$ to obtain

$$
4 \pi \int_{\mathcal{C}} \rho=\int_{\partial \mathcal{T}}\left(\star d f^{-1}+\frac{8}{9} C \wedge G^{+}\right)
$$

where $C$ is a potential for $G$ in $\mathcal{T}$, i.e., $G=d C$. The right hand side is sometimes called the Page charge. It is obviously gauge invariant under $C \rightarrow C+d \lambda$. However, if $\mathcal{C}$ is a closed loop then $\mathcal{T}$ has non-trivial first cohomology and hence there is the freedom to perform large gauge transformations $C \rightarrow C+\theta$ where $\theta$ is closed but not exact. Therefore the above expression is not gauge-invariant. See [19] for further discussion of this issue. In the present case we can resolve this ambiguity as follows. First we can define a conserved charge by

$$
\mathrm{Q}=\frac{1}{4 \pi} \int_{S^{3}} \star d f^{-1}
$$

where the $S^{3}$ is a sphere at infinity in $R^{4}$. One can check that this is proportional to the usual definition of the electric charge as the integral of $\star_{5} F$ over $S^{3}$ where $\star_{5}$ denotes the five-dimensional Hodge dual. Now choose a gauge in which $C$ is globally defined on $R^{4}-\mathcal{C}$ and vanishes at infinity (for example, the gauge in which $C$ is given by the usual Green's function integral in $R^{4}$ ). Equation (2.9) can now be written ${ }^{3}$

$$
d\left(\star d f^{-1}+\frac{8}{9} C \wedge G^{+}\right)=4 \pi \rho \delta[\mathcal{C}] \star 1 .
$$

Integrating this equation over $R^{4}$ we then obtain

$$
\mathbf{Q}=\int_{\mathcal{C}} \rho
$$

where we have used the fact that $C$ and $G^{+}$vanish at infinity. Hence the total charge is determined by the integral of the charge density of the source, just as expected physically.

\footnotetext{
${ }^{3}$ Actually these equations are not the same but differ by a term proportional to $C \wedge \star J$ supported on $\mathcal{C}$. However, this term is not well-defined because $C$ is singular on $\mathcal{C}$. In practice we must adopt (2.12) as our definition of $\rho$ since we need to be able to integrate the LHS in order to make sense of the delta function on the RHS.
} 
Note also that by integrating the LHS of (2.12) over $R^{4}-\mathcal{T}$ we conclude that the RHS of (2.10) is gauge invariant (as it is just $4 \pi \mathrm{Q}$ ) provided we demand that $C$ be well-defined on $R^{4}-\mathcal{C}$, not just on $\mathcal{T}$. This is possible because $R^{4}-\mathcal{C}$ has vanishing first cohomology so demanding that $C$ be globally defined excludes large gauge transformations.

The final subtlety concerns how one uses (2.12) to calculate the local charge density $\rho$ (rather than the integral of $\rho$ ). Pick a point $p$ on $\mathcal{C}$. Choose some surface $S(p)$ that intersects $\mathcal{C}$ orthogonally at $p$. Let $n$ denote the unit normal to $S(p)$ and $B(p, \epsilon)$ a 3-ball in $S(p)$ centered on $p$ with radius $\epsilon$. If we contract (2.12) with $n$ and integrate over $B(p, \epsilon)$ we obtain

$$
\rho(p)=\frac{1}{4 \pi} \int_{B(p, \epsilon)} i_{n} d Y
$$

where $i_{n}$ denotes the operation of contracting $n$ with the first index of a form, and

$$
Y \equiv \star d f^{-1}+\frac{8}{9} C \wedge G^{+}
$$

A short calculation gives

$$
\left(i_{n} d Y\right)_{\mu \nu \rho}=n \cdot \nabla Y_{\mu \nu \rho}+\left[d\left(i_{n} Y\right)\right]_{\mu \nu \rho}-3 K_{[\mu}^{\sigma} Y_{\nu \rho] \sigma}+\ldots
$$

where $K_{\mu \nu}$ is the extrinsic curvature of $S(p)$ and the ellipsis denotes a term that vanishes when projected onto $S(p)$. Substituting into (2.14) we get

$$
\rho(p)=\frac{1}{4 \pi} \int_{\partial B(p, \epsilon)} i_{n} Y+\text { volume terms. }
$$

If we now take $\epsilon \rightarrow 0$ then the volume contributions vanish (this can be checked explicitly using the solutions we shall present later) leaving

$$
\rho(p)=\frac{1}{4 \pi} \lim _{\epsilon \rightarrow 0} \int_{\partial B(p, \epsilon)} i_{n}\left(\star d f^{-1}+\frac{8}{9} C \wedge G^{+}\right) .
$$

As an example, let's calculate $\rho$ for the black ring solution of [6]. Following the notation of that paper, $\mathcal{C}$ is a circle of radius $R$. We take $C=(3 q / 4)(1+y) d \psi$. This satisfies the above requirement of being well-defined on $R^{4}-\mathcal{C}$ and vanishing at infinity. If the point $p$ has coordinate $\psi$ then we define $S(p)$ to be a surface of constant $\psi$ through $p$. We then choose $B(p, \epsilon)$ to be the region $y<-1 / \epsilon$ of $S(p)$. Hence $\partial B(p, \epsilon)$ is an $S^{2}$ parametrized by $(x, \phi)$. We have $n=(1 / R)[1+\mathcal{O}(\epsilon)] \partial / \partial \psi$ and find that $Y_{\psi x \phi}=(Q / 2)-q^{2} x+\mathcal{O}(\epsilon)$. Plugging these results into (2.18) then gives $\rho=Q /(2 R)$, i.e., the charge density is constant and proportional to $Q$. There is no explicit contribution from the fluxes ${ }^{4}$.

\footnotetext{
${ }^{4}$ One manifestation of the confusion in [7] is that, in their discussion of this solution (setting their three charges equal to obtain a solution of minimal 5D supergravity), BW claim (after equation (42)) that the coefficient of $x-y$ in their function $Z$ is proportional to the charge density $\rho$. Our calculation using equation (2.18) reveals that this is incorrect.
} 
Returning to the original task of solving the supergravity equations, we still need to determine $\omega$. The latter is not gauge invariant: a shift $t \rightarrow t^{\prime}=t-\lambda(x)$ gives $\omega \rightarrow \omega^{\prime}=$ $\omega+d \lambda$. It is therefore convenient to work with the field strength $W=d \omega$. From the definition of $G^{+}$we see that $W$ must obey

$$
d W=0, \quad W+\star W=2 f^{-1} G^{+}
$$

BW showed how to solve these equations to determine $W$ up to the addition of a closed, anti-self-dual two-form. In practice, one can attempt to use this freedom to ensure that $\omega$ is globally defined and that the resulting solution is free of closed timelike curves.

\section{$3 \quad$ Straight string}

In this section we shall consider a solution describing an infinite string, in which the curve $\mathcal{C}$ is taken to be a straight line in flat space with the charge density allowed to vary along the line. Such solutions were briefly discussed in [10], but not given explicitly. If the charge density is given by a periodic function then one can identify the solution to obtain a finite string wrapped on a Kaluza-Klein circle. We shall show that this solution has a smooth horizon only if the charge density is constant.

\subsection{The solution}

Take $\mathcal{C}$ to be a straight line $L$. Choose cylindrical polar coordinates centered on $L$ :

$$
h_{m n} d x^{m} d x^{n}=d z^{2}+d r^{2}+r^{2}\left(d \theta^{2}+\sin ^{2} \theta d \phi^{2}\right) .
$$

Let $J$ be a constant density line source on $L$ :

$$
J=-3 \pi q d z \delta[L] .
$$

A solution for $C$ is

$$
C=-\frac{3 q}{4 r} d z,
$$

which is well-defined on $R^{4}-L$ and vanishes at infinity, as required. The solution for $G^{+}$is then

$$
G^{+}=-\frac{3 q}{4 r^{2}}\left(d z \wedge d r+r^{2} \sin \theta d \theta \wedge d \phi\right),
$$

where we have chosen the orientation $\epsilon_{z r \theta \phi}=+1$. Equation (2.4) is

$$
\nabla^{2} f^{-1}=\frac{q^{2}}{2 r^{4}}
$$


with solution

$$
f(r, z)^{-1}=\frac{q^{2}}{4 r^{2}}+\int_{-\infty}^{\infty} \frac{\rho\left(z^{\prime}\right) d z^{\prime}}{\pi\left(r^{2}+\left(z-z^{\prime}\right)^{2}\right)}+1
$$

where we have normalized $f=1$ at $r=\infty$ and allowed for a varying charge density $\rho(z)$ on $L{ }^{5}$ Finally we can solve for $\omega$ starting from the ansatz

$$
\omega=w(r, z) d z
$$

which leads to

$$
\partial_{r} w=\frac{3 q^{3}}{8 r^{4}}+\frac{3 q}{2 r^{2}} \int_{-\infty}^{\infty} \frac{\rho\left(z^{\prime}\right) d z^{\prime}}{\pi\left(r^{2}+\left(z-z^{\prime}\right)^{2}\right)}+\frac{3 q}{2 r^{2}} .
$$

The integral can be computed if we assume that

$$
\rho(z)=\operatorname{Re} F(z)
$$

where $F(z)$ is a holomorphic function with suitable behaviour as $|z| \rightarrow \infty$ with $\operatorname{Im} z>0$, and no poles in the upper half-plane (e.g. $F(z)=a+b \exp (i z))$ :

$$
\int_{-\infty}^{\infty} \frac{\rho\left(z^{\prime}\right) d z^{\prime}}{\pi\left(r^{2}+\left(z-z^{\prime}\right)^{2}\right)}=\frac{1}{r} \operatorname{Re} F(z+i r) .
$$

We can then expand in $r$ for small $r$ :

$$
\begin{gathered}
f^{-1}=\frac{q^{2}}{4 r^{2}}+\frac{1}{r} \operatorname{Re} F(z)+1-\operatorname{Im} F^{\prime}(z)-\frac{r}{2} \operatorname{Re} F^{\prime \prime}(z)+\mathcal{O}\left(r^{2}\right) \\
w=-\frac{q^{3}}{8 r^{3}}-\frac{3 q}{4 r^{2}} \operatorname{Re} F(z)-\frac{3 q}{2 r}\left(1-\operatorname{Im} F^{\prime}(z)\right)-\frac{3 q}{4} \operatorname{Re} F^{\prime \prime}(z) \log r+\mathcal{O}(r) .
\end{gathered}
$$

The $\log r$ term can be eliminated by a gauge transformation $t \rightarrow t^{\prime}=t-\lambda(x)$ at the expense of introducing a $r$-component into $\omega$.

\subsection{Geometry near the source}

We now examine the metric near $L$. We have

$$
\begin{gathered}
g_{t t}=-f^{2}=\mathcal{O}\left(r^{4}\right), \\
g_{t z}=-f^{2} w=\frac{2 r}{q}+\mathcal{O}\left(r^{2}\right), \\
g_{z z}=f^{-1}-f^{2} w^{2}=\frac{p(z)}{q^{2}}+\mathcal{O}(r \log r),
\end{gathered}
$$

\footnotetext{
${ }^{5}$ Equation (2.18) can be used to verify the $\rho$-dependence of this expression. Choose $S(p)$ to be the plane with the same $z$-coordinate as $p$ and $B(p, \epsilon)$ to be the ball $r<\epsilon$ on $S(p)$.
} 
where

$$
p(z) \equiv 3\left[(\operatorname{Re} F(z))^{2}+\left(\operatorname{Im} F^{\prime}(z)-1\right) q^{2}\right] .
$$

We shall assume that $F(z)$ is chosen so that $p(z)$ is positive. The leading order metric near $r=0$ is

$$
d s^{2}=\frac{4 r}{q} d t d z+\frac{p(z)}{q^{2}} d z^{2}+\frac{q^{2}}{4} \frac{d r^{2}}{r^{2}}+\frac{q^{2}}{4} d \Omega^{2} .
$$

On a constant $t$ surface, the horizon appears to be at $r=0$, and $z$ appears to be a good coordinate there. To see that this is incorrect, consider first the special case $p=$ constant. Using the symmetries, one can easily check that all geodesics that approach $r=0$ have $|z|$ diverge. To construct good coordinates, let

$$
v=t-\frac{q \sqrt{p}}{4 r}, \quad \hat{z}=z-\frac{q^{2}}{2 \sqrt{p}} \log (r / q),
$$

to get

$$
d s^{2}=\frac{4 r}{q} d v d \hat{z}+\frac{2 q}{\sqrt{p}} d v d r+\frac{p}{q^{2}} d \hat{z}^{2}+\frac{q^{2}}{4} d \Omega^{2},
$$

which defines an analytic extension through a future horizon at $r=0$. Note that $\hat{z}$ is a good coordinate on the horizon, which implies that $z \rightarrow-\infty$ there.

Now return to the general case of varying $p$. This metric is very similar to the one studied in [14. As shown there, the metric is equivalent to $A d S_{3} \times S^{2}$. Let $\sigma$ be a solution to

$$
\sigma^{\prime}(z)+\sigma(z)^{2}=\frac{p(z)}{q^{4}}
$$

and set $\log H(z)=\int^{z} \sigma$. Introducing new coordinates

$$
U=\int \frac{d z}{H(z)^{2}}, \quad V=\frac{2 t}{q^{4}}+\frac{\sigma(z)}{2 q r}, \quad Z=\sqrt{2 q r} H(z),
$$

the metric (3.17) becomes

$$
d s^{2}=q^{2}\left[Z^{2} d U d V+\frac{d Z^{2}}{Z^{2}}\right]+\frac{q^{2}}{4} d \Omega^{2},
$$

the product of an $\mathrm{AdS}_{3}$ space of radius $q$ with an $S^{2}$ of radius $q / 2$. To understand where the horizon is in these coordinates, we reconsider the case of constant $p$. We choose $\sigma=-\sqrt{p} / q^{2}$. Then writing $(U, V, Z)$ in terms of $(r, v, \hat{z})$ gives $H(z)=\exp \left(-z \sqrt{p} / q^{2}\right)$ and

$$
U \propto r \exp \left(\frac{2 \sqrt{p}}{q^{2}} \hat{z}\right), \quad V \propto v, \quad Z \propto \exp \left(-\frac{\sqrt{p}}{q^{2}} \hat{z}\right) \quad \text { (constant p) }
$$

The horizon is located at $r=0$ with finite $v$ and $\hat{z}$ and hence at $U=0$ with finite $V$ and (non-zero) $Z$. In the case of non-constant $p$, this implies that $H$, and hence $z$, must diverge at the horizon. Hence a point on $L$ does not correspond to a point on the $A d S_{3}$ horizon. 
We have shown that the leading order metric (3.17) has a smooth horizon at $r=0$. In fact, this horizon is completely homogeneous, even when $p(z)$ is not constant. It does not retain any information about the varying charge distribution. This horizon is also infinite in extent. However, we can periodically identify the coordinate $z$ or, equivalently, take $\rho(z)$ to be a periodic function. The leading order metric then has a smooth finite horizon. ${ }^{6}$ However, this does not imply that the full solution has a smooth horizon. The subleading terms in the metric play a crucial role. We will now show that these subleading terms behave so badly that the full metric does not admit a $C^{2}$ horizon.

The metric has an $O(3)$ symmetry with $S^{2}$ orbits. Let $\mathcal{A}$ denote the area of the $S^{2}$ through any point. If the metric had a $C^{2}$ (or even $C^{1}$ ) horizon, then the first derivative of $\mathcal{A}$ off the horizon would have to be continuous. But since

$$
\mathcal{A}=4 \pi r^{2} f^{-1}
$$

we have

$$
\left.d \mathcal{A}\right|_{r=0}=4 \pi \rho(z) d r
$$

so $\rho(z)$ would have to be continuous at the horizon. But we have seen above that $z$ diverges at a horizon. Hence the existence of a horizon implies that $\rho$ must have a well-defined limit as $z$ diverges. This is impossible if $\rho(z)$ is a non-trivial periodic function. Hence the only solution with a compact $C^{2}$ horizon is the one with constant $\rho$.

There is a slight loophole in this argument since we have not shown that $d r$ is nonvanishing at the horizon when $\rho(z)$ is not constant. A more rigorous argument goes as follows. If the solution is $C^{2}$ then the connection is $C^{1}$ and the Killing spinor should be $C^{2}$. $f$ must also be $C^{2}$ because it is the square of the Killing spinor [18]. From [20] we know that $f$ has a second order zero at the horizon for any solution with an $A d S_{3} \times S^{2}$ near-horizon geometry. The explicit form of the solution is

$$
f=\frac{4 r^{2}}{q^{2}}+\mathcal{O}\left(r^{3}\right) .
$$

It follows that $r$ is $C^{1}$ with a first-order zero at the horizon. Hence $d r$ is $C^{0}$ and non-vanishing at the horizon.

\section{Arbitrary curves}

In this section we consider the solution associated with an arbitrary closed curve in $R^{4}$. We first introduce a convenient coordinate system, then find the solution near the horizon (including the subleading contribution), and finally show that the horizon cannot be $C^{2}$.

\footnotetext{
${ }^{6}$ This assumes that a periodic solution to (3.20) exists. An argument for this was given in [14].
} 


\subsection{Fermi normal coordinates}

Consider a curve $\mathcal{C}$ in $R^{4}$ given by $x=x(\ell)$ where $\ell$ denotes proper length along the curve (so $\left.\dot{x}^{2}=1\right)$. We are primarily interested in a closed curve without self-intersections so we shall assume that $\ell$ is a periodic coordinate. Let $e_{i}(\ell)$ be three orthonormal vectors defined along the curve that are normal to the curve (i.e. $\left.\dot{x}(\ell) \cdot e_{i}(\ell)=0\right)$. Assign Fermi normal coordinates $\left(\ell, X^{i}\right)$ to the point a distance $|\mathbf{X}|$ along the straight line from $x(\ell)$ in the direction $X^{i} e_{i}(\ell)$. These coordinates are related to Cartesian coordinates by

$$
x=x(\ell)+X^{i} e_{i}(\ell) .
$$

There is a natural choice of basis $e_{i}$ given by $(a, b, \ldots$ denote Cartesian coordinates)

$$
e_{i}^{a}(\ell)=\dot{x}^{b}(\ell) J_{b}^{(i) a}
$$

where $J^{(i)}$ are the three anti-self-dual complex structures:

$$
J^{(1)}=d x^{1} \wedge d x^{2}-d x^{3} \wedge d x^{4}, \quad J^{(2)}=d x^{1} \wedge d x^{3}+d x^{2} \wedge d x^{4}, \quad J^{(3)}=d x^{1} \wedge d x^{4}-d x^{2} \wedge d x^{3}
$$

This gives

$$
\dot{x} \cdot \dot{e}_{i}(\ell)=-a_{i}(\ell), \quad e_{i}(\ell) \cdot \dot{e}_{j}(\ell)=-\epsilon_{i j k} a_{k}(\ell), \quad \dot{e}_{i}(\ell) \cdot \dot{e}_{j}(\ell)=\delta_{i j} \mathbf{a}(\ell)^{2}
$$

where $a \equiv \ddot{x}$ is the acceleration of the curve, and $a_{i}(\ell) \equiv a(\ell) \cdot e_{i}(\ell)$. We shall regard $a_{i}$ as the components of a 3 -vector a in $R^{3}$ with coordinates $X^{i}$. The second of these equations says that this basis has rotation equal to minus the acceleration of the curve. In Fermi coordinates, the flat metric on $R^{4}$ is

$$
\begin{aligned}
d s^{2} & =\left(1-2 \mathbf{a}(\ell) \cdot \mathbf{X}+\mathbf{a}(\ell)^{2} \mathbf{X}^{2}\right) d \ell^{2}+2(\mathbf{a}(\ell) \times \mathbf{X})_{i} d \ell d X^{i}+\delta_{i j} d X^{i} d X^{j} \\
& =(1-\mathbf{a}(\ell) \cdot \mathbf{X})^{2} d \ell^{2}+(d \mathbf{X}+\mathbf{a}(\ell) \times \mathbf{X} d \ell)^{2}
\end{aligned}
$$

where $\mathbf{a} \cdot \mathbf{X} \equiv a_{i} X^{i}$ and $(\mathbf{a} \times \mathbf{X})_{i} \equiv \epsilon_{i j k} a_{j} X^{k}$. Hence $\sqrt{h}=1-\mathbf{a}(\ell) \cdot \mathbf{X}$. These coordinates are not valid globally but are well defined in a finite neighborhood of the original curve. Note that (by construction) the surfaces of constant $\ell$ are flat. Everything above can be generalized to an arbitrary base space (the metric then contains terms quadratic in $\mathbf{X}$ that involve the curvature).

As an example, consider a curve that is confined to the 12-plane:

$$
x^{1}=x^{1}(\ell), \quad x^{2}=x^{2}(\ell), \quad x^{3}=x^{4}=0 .
$$

Using (4.3), the basis vectors are

$$
e_{1}=\left(-\dot{x}^{2}, \dot{x}^{1}, 0,0\right), \quad e_{2}=\left(0,0, \dot{x}^{1}, \dot{x}^{2}\right), \quad e_{3}=\left(0,0,-\dot{x}^{2}, \dot{x}^{1}\right) .
$$


The Cartesian coordinates are related to the Fermi coordinates by

$$
\begin{aligned}
& x^{1}=x^{1}(\ell)-X^{1} \dot{x}^{2}(\ell), \quad x^{2}=x^{2}(\ell)+X^{1} \dot{x}^{1}(\ell) \\
& x^{3}=X^{2} \dot{x}^{1}(\ell)-X^{3} \dot{x}^{2}(\ell), \quad x^{4}=X^{2} \dot{x}^{2}(\ell)+X^{3} \dot{x}^{1}(\ell) .
\end{aligned}
$$

Note that the curve (4.6) is invariant under the flow of the vector field

$$
x^{3} \frac{\partial}{\partial x^{4}}-x^{4} \frac{\partial}{\partial x^{3}}=X^{2} \frac{\partial}{\partial X^{3}}-X^{3} \frac{\partial}{\partial X^{2}},
$$

and this vector field will consequently generate a symmetry of the resulting solution, i.e., it will be a Killing vector field. The components of the acceleration are

$$
a_{1}=\dot{x}^{1} \ddot{x}^{2}-\dot{x}^{2} \ddot{x}^{1}, \quad a_{2}=a_{3}=0
$$

For a circle of radius $R, x^{1}=R \cos (\ell / R)$ and $x^{2}=R \sin (\ell / R)$ so $a_{1}=1 / R$ independent of $\ell$. If the charge density is constant then this solution is the solution of [6] with a regular $S^{1} \times S^{2}$ horizon. In this case, the coordinates $X^{i}$ are related to the ring coordinates $(\theta, \chi)$ regular on the $S^{2}$ of the horizon by $X^{1}=r \cos \theta, X^{2}=r \sin \theta \cos \chi, X^{3}=r \sin \theta \sin \chi$ hence $\mathbf{X} /|\mathbf{X}|$ is smooth on the $S^{2}$ of the horizon and the Killing field (4.9) is $\partial / \partial \chi$. In this example, $\partial / \partial \ell$ is also a Killing field.

\subsection{Solving the equations}

Consider a source of constant density along the curve:

$$
J=-3 \pi q \dot{x}(\ell) \delta[\mathcal{C}]
$$

Writing $G=d C$, the solution for $C$ in Cartesian coordinates is

$$
C(x)=-\frac{3 q}{4 \pi} \int \frac{\dot{x}(\tilde{\ell}) d \tilde{\ell}}{[x-x(\tilde{\ell})]^{2}}
$$

and hence

$$
G(x)=\frac{3 q}{2 \pi} \int \frac{[x-x(\tilde{\ell})] \wedge \dot{x}(\tilde{\ell}) d \tilde{\ell}}{[x-x(\tilde{\ell})]^{4}}
$$

To evaluate this integral, we first write the field point $x$ in terms of Fermi normal coordinates $\left(\ell, X^{i}\right)$ using (4.1) and expand

$$
x(\tilde{\ell})=x(\ell)+\dot{x}(\ell)(\tilde{\ell}-\ell)+\frac{1}{2} \ddot{x}(\ell)(\tilde{\ell}-\ell)^{2}+\ldots
$$


Then

$$
[x-x(\tilde{\ell})]^{2}=\mathbf{X}^{2}+(1-\mathbf{a} \cdot \mathbf{X})(\tilde{\ell}-\ell)^{2}+\ldots
$$

Setting $\tilde{\ell}=\ell+|\mathbf{X}| \eta$, one can expand the integral for small $|\mathbf{X}|$ to obtain

$$
G(\ell, \mathbf{X})=-\frac{3 q}{4|\mathbf{X}|^{3}} \dot{x}(\ell, \mathbf{X}) \wedge e_{i}(\ell, \mathbf{X})\left(X^{i}+\frac{1}{2} X^{i} X^{j} a_{j}(\ell)+\frac{1}{2} \mathbf{X}^{2} a_{i}(\ell)\right)+\mathcal{O}(1)
$$

In this expression, the orthonormal basis $\left(\dot{x}(\ell), e_{i}(\ell)\right)$ defined along the curve is extended to a global orthonormal basis $\left(\dot{x}(\ell, \mathbf{X}), e_{i}(\ell, \mathbf{X})\right)$ by translating along the (flat) surfaces of constant $\ell$. Now we need to rewrite this basis in the Fermi coordinates. Using equations (4.1) and (4.4) we obtain

$$
\dot{x}(\ell, \mathbf{X})=\frac{1}{1-\mathbf{a} \cdot \mathbf{X}}\left[\frac{\partial}{\partial \ell}-(\mathbf{a} \times \mathbf{X})_{i} \frac{\partial}{\partial X^{i}}\right], \quad e_{i}(\ell, \mathbf{X})=\frac{\partial}{\partial X^{i}},
$$

or, in terms of covectors:

$$
\dot{x}(\ell, \mathbf{X})=(1-\mathbf{a} \cdot \mathbf{X}) d \ell, \quad e_{i}(\ell, \mathbf{X})=d X^{i}+(\mathbf{a} \times \mathbf{X})_{i} d \ell .
$$

Hence we have

$$
G=-\frac{3 q}{4|\mathbf{X}|^{3}} d \ell \wedge\left(X^{i}-\frac{1}{2} X^{j} a_{j}(\ell) X^{i}+\frac{1}{2} \mathbf{X}^{2} a_{i}(\ell)\right) d X^{i}+\mathcal{O}(1) .
$$

Choosing orientation $\epsilon_{\ell i j k}=\epsilon_{i j k}$ we then get

$$
\begin{aligned}
G^{+} & =-\frac{3 q}{4|\mathbf{X}|^{3}}\left[\left(X^{i}-\frac{3}{2} X^{j} a_{j}(\ell) X^{i}+\frac{3}{2} \mathbf{X}^{2} a_{i}(\ell)\right) d \ell \wedge d X^{i}\right. \\
& \left.+\frac{1}{2} \epsilon_{i j k}\left(X^{k}+\frac{1}{2} X^{j} a_{j}(\ell) X^{k}+\frac{1}{2} \mathbf{X}^{2} a_{k}(\ell)\right) d X^{i} \wedge d X^{j}\right]+\mathcal{O}(1) .
\end{aligned}
$$

Note that the leading terms are independent of the acceleration of the curve and agree with the solution for a straight string.

Now we determine $f$ by solving

$$
\Delta f^{-1}=\frac{4}{9}\left(G^{+}\right)^{2}=\frac{q^{2}}{2|\mathbf{X}|^{4}}(1+2 \mathbf{a} \cdot \mathbf{X})+\mathcal{O}\left(1 / \mathbf{X}^{2}\right) .
$$

The boundary condition is that $f \rightarrow 1$ at infinity in $R^{4}$ but this is not required to extract the singular small $\mathbf{X}$ behavior of $f^{-1}$ which can be done using the method of section 2. The result is

$$
f^{-1}=\frac{q^{2}}{4 \mathbf{X}^{2}}(1-\mathbf{a}(\ell) \cdot \mathbf{X})+\frac{b(\ell)}{|\mathbf{X}|}+\mathcal{O}(1) .
$$

The $1 /|\mathbf{X}|$ term arises from the freedom to add a harmonic function to $f^{-1}$ so that (2.12) is satisfied with charge density $\rho(\ell)$. The explicit relationship between $b$ and $\rho$ is easy to obtain 
from equation (2.18) for the case in which $\mathcal{C}$ is a circle of radius $R$ because we already know the full solution for $C$ and $G^{+}$in that case (see the discussion below equation (2.18) ). We find that

$$
b(\ell)=\rho(\ell)-q^{2} /(2 R) \quad(\mathcal{C} \text { a circle of radius } R) .
$$

Unfortunately, we have not been able to obtain a simple result expressing $b(\ell)$ in terms of $\rho(\ell)$ for a general curve $\mathcal{C}$. The problem is that in order to compute $\rho$ using (2.18) it is necessary to expand the integral (4.12) for $C$ to second non-trivial order in $\mathbf{X}$. In particular, we need to know the $\mathbf{X}$-independent part of the $\ell$ component of $C$. There does not appear to be a local expression for this term for a general curve $\mathcal{C}$ so all we can conclude is that $b(\ell)=\rho(\ell)-c(\ell)$ for some function $c$ that depends only on the curve $\mathcal{C}$.

The final step is to calculate $\omega$. The leading order solution for $\omega$ can be obtained from the straight string. The next order is $\mathcal{O}\left(1 / \mathbf{X}^{2}\right)$, where we will find terms linear in $\mathbf{a}$ or $b$. The $b$-dependent correction is independent of a and hence should be the same as for the straight string. To calculate the a dependent corrections, we note that the only possible $\mathcal{O}\left(1 / \mathbf{X}^{2}\right)$ term in $\omega_{\ell}$ is proportional to $\mathbf{a} \cdot \mathbf{X} /|\mathbf{X}|^{3}$. In $\omega_{i}$ there are three possibilities, proportional to $(\mathbf{a} \times \mathbf{X})_{i} /|\mathbf{X}|^{3}, a_{i} / \mathbf{X}^{2}$ and $\mathbf{a} \cdot \mathbf{X} X^{i} /|\mathbf{X}|^{4}$ respectively. Plugging a general linear combination of these terms into equation (2.2), we find that the final two terms appear with coefficients such that they combine into a total derivative, which can be eliminated by shifting in the time coordinate $t$. This leaves

$$
\omega=-\frac{q^{3}}{8|\mathbf{X}|^{3}}\left[\left(1-\frac{3}{2} \lambda \mathbf{a} \cdot \mathbf{X}\right) d \ell-\frac{3}{2}(1-\lambda)(\mathbf{a} \times \mathbf{X})_{i} d X^{i}\right]-\frac{3 q b}{4 \mathbf{X}^{2}} d \ell+\mathcal{O}(1 /|\mathbf{X}|),
$$

where $\lambda$ is an arbitrary constant related to the freedom to add a closed anti-self-dual term to $d \omega$. We determine $\lambda$ by noting that the $\ell i$ and $\ell \ell$ components of the five-dimensional metric diverge at $\mathbf{X}=0$ unless we choose $\lambda=5 / 3$. Therefore we must make this choice to ensure that $\mathcal{C}$ has finite proper circumference.

\subsection{Absence of a $C^{2}$ horizon}

If the above solution admits a smooth horizon then a result of [20] establishes that the nearhorizon geometry must be $A d S_{3} \times S^{2}$. We have seen already how this geometry arises as the leading order metric of a straight string. Now we shall see how it arises for a general curve $\mathcal{C}$. First we need to be more precise about what we mean by "leading order metric". Obviously this must involve a limit $\mathbf{X} \rightarrow 0$ so we start by rescaling $\mathbf{X} \rightarrow \epsilon \mathbf{X}$. To keep the metric non-degenerate as we send $\epsilon \rightarrow 0$ we must also rescale $t \rightarrow t / \epsilon$ (so $t$ diverges as $\epsilon \rightarrow 0$, as expected at a horizon). The resulting metric is

$$
d s^{2}=\frac{4 r}{q} d t d \ell+\frac{p(\ell, \theta, \chi)}{q^{2}} d \ell^{2}+\frac{q^{2}}{4} \frac{d r^{2}}{r^{2}}+\frac{q^{2}}{4}\left(d \theta^{2}+\sin ^{2} \theta d \chi^{2}\right) .
$$


where we have parametrized $\mathbf{X}$ with spherical polar coordinates $(r, \theta, \chi)$ and $p(\ell, \theta, \chi)$ is a function that cannot be determined just from the leading order form of the solution presented above - one would have to go to third order in $|\mathbf{X}|$ to calculate it, just as for the straight string. However, it turns out that this does not matter. Since we know that the above metric has to be $A d S_{3} \times S^{2}$, it is easy to show that $p$ must be independent of $\theta$ and $\chi \cdot{ }^{7}$ The above metric is then the same as (3.17) (with $z \rightarrow \ell$ ) and hence manifestly $A d S_{3} \times S^{2}$ with a smooth horizon at $r=0$ at which $\ell$ diverges. We can now use this fact to deduce that the full metric does not have a smooth horizon in general.

In order to argue that the solution does not admit a $C^{2}$ horizon, we shall repeat the strategy used for the straight string. We shall assume that there is a $C^{2}$ horizon and arrive at a contradiction by constructing a scalar whose gradient is not continuous at the horizon. Since the solution is no longer spherically symmetric, we cannot use the area of two-spheres as we did for the straight string. Instead, consider

$$
f^{2}\left(G^{+}\right)^{2}=\frac{18}{q^{2}}\left(1+4 \mathbf{a}(\ell) \cdot \mathbf{X}-\frac{8 b(\ell)}{q^{2}}|\mathbf{X}|\right)+\mathcal{O}\left(\mathbf{X}^{2}\right) .
$$

The left hand side is a scalar invariant of the solution. The analysis of [20] reveals that it should be at least $C^{1}$ at the horizon. Writing $\mathbf{X}=r \hat{\mathbf{X}}$, we obtain

$$
d\left[f^{2}\left(G^{+}\right)^{2}\right]=\frac{72}{q^{2}}\left(\mathbf{a}(\ell) \cdot \hat{\mathbf{X}}-\frac{2 b(\ell)}{q^{2}}\right) d r
$$

at $r=0$. The left hand side should be continuous at the horizon. As before, we can now use the fact that, if the solution is $C^{2}$, then $f$ is $C^{2}$ with a second order zero on the horizon. This implies that $r \equiv|\mathbf{X}|$ is $C^{1}$ with a first order zero at the horizon so $d r$ is continuous and non-vanishing there. From the leading order metric, we know that $\theta$ and $\chi$ are continuous at the horizon, and hence so is $\hat{\mathbf{X}}$. Since $\ell$ diverges at the horizon, the only way that the right hand side can be continuous is if the periodic functions a and $b$ are independent of $\ell$. Existence of a $C^{2}$ horizon therefore implies that $\mathbf{a}$ and $b$ must be independent of $\ell$. Note that $b(\ell)$ cannot cancel the acceleration dependence since the acceleration term is direction dependent.

We have concluded that existence of a $C^{2}$ horizon requires that $b$ and a be independent of $\ell$. The latter requirement is equivalent to

$$
0=\frac{d a_{i}}{d \ell}=\frac{d^{3} x^{a}}{d \ell^{3}} \frac{d x^{b}}{d \ell} J_{a b}^{(i)}
$$

\footnotetext{
${ }^{7}$ By calculating the eigenvalues of $R_{\nu}^{\mu}$ one can deduce that $\partial / \partial \theta$ and $\partial / \partial \chi$ must be tangent to the $S^{2}$ of $A d S_{3} \times S^{2}$. It then follows from the above metric that $\partial / \partial t, \partial / \partial \ell$ and $\partial / \partial r$ must be tangent to $A d S_{3}$. This implies that the Riemann tensor must factorize in the above coordinate system. This implies $\partial_{\theta} p=\partial_{\chi} p=0$.
} 
If a two-form has vanishing contraction with all of the $J^{(i)}$ then it must be self-dual. Any non-zero self-dual form has rank four. However, the two-form above clearly has rank two. It must therefore vanish. Hence we must have

$$
\frac{d^{3} x^{a}}{d \ell^{3}} \propto \frac{d x^{a}}{d \ell}
$$

The coefficient of proportionality probably has to be constant since otherwise we can construct a $\ell$-dependent scalar by taking the dot product of the LHS and RHS, and this scalar will probably appear in the expansion of the solution at next order and lead to a lack of smoothness at the horizon. Hence, after a shift in $x^{a}$, we get

$$
\frac{d^{2} x^{a}}{d \ell^{2}} \pm \frac{x^{a}}{R^{2}}=0
$$

for some constant $R$, with solutions given by straight lines, circles or hyperbolae. However, the latter do not satisfy the normalization condition $\dot{x}^{2}=1$ so must be excluded. The circles always lie in a 2-plane.

Note that it is not sufficient for the curve to follow a symmetry of the base space metric. For flat $R^{4}$, a helical curve has a tangent vector which is a linear combination of a rotation and a translation, and hence is a Killing field. Nevertheless, this curve does not satisfy (4.29). The problem is that even though $\ddot{x}$ is not affected by the added translation, the basis vectors $e_{i}$ are, so the components of the acceleration $a_{i}$ pick up extra $\ell$ dependence.

To summarize, modulo a plausible assumption about the coefficient of proportionality in (4.29), we have shown that the only asymptotically flat solutions with a $C^{2}$ horizon are those for which $\mathcal{C}$ is a circle and $b(\ell)$ is constant. Since we know the precise relationship between $b$ and the charge density $\rho$ for circular $\mathcal{C}$ (equation (4.23)), we can conclude that the charge density $\rho$ is constant. So it seems that the only solution with a $C^{2}$ horizon is the black ring of [6] (and in that case, the horizon is actually smooth).

\section{Discussion}

Recently, Bena, Wang and Warner have constructed some explicit solutions describing a circular ring with three varying charge densities [21. They show that one can adjust the charge densities so that the function $p(\ell)$ in the near horizon metric (4.25) is constant. Since this is only one constraint on three functions, there are still two free functions worth of freedom. However we have seen that a constant $p(\ell)$ is not necessary for the leading order horizon metric to be smooth, and not sufficient to ensure that the full metric is smooth at the horizon. Even though their metrics are not solutions to minimal five-dimensional supergravity, the argument given in the previous section is easily extended to show that 
their solutions do not have smooth horizons. Their solutions contain extra scalars which can be viewed as the radii of the internal circles in a toroidal compactification of M-theory. Derivatives of these scalars off the horizon depend on $\ell$ and will not be continuous on the horizon.

We have focussed on the question of smoothness of the horizon since this is crucial for the no hair theorems in classical general relativity. The fact that most of the solutions described in [7] do not have smooth horizons does not mean that they have no physical interest. In the analogous situation of traveling waves along extremal black strings, even though the curvature diverges at the horizon, the metric is continuous there so the horizon area is well defined. It turns out that this area depends on the wave profile and one can reproduce the entropy of the black string by counting string states with the prescribed wave profile [14]. It would be interesting to know if the metric of the BW solutions is also continuous at the horizon. One might think that this is obvious since we have shown that the leading order metric near the horizon is locally $A d S_{3} \times S^{2}$. However this only shows that the leading order divergences in the metric are purely coordinate effects. Any subleading divergences still remain to be addressed. The entropy of the $U(1)^{2}$ invariant supersymmetric black rings of [6, 7, 8, 9] has recently been reproduced by a microscopic counting of states in M theory 22] (see 23] for an earlier discussion of this problem). If the more general solutions turn out to have a continuous horizon with well defined area, one could try to extend this argument to reproduce their entropy.

\section{Acknowledgments}

It is a pleasure to thank H. Elvang, R. Emparan, D. Marolf and D. Mateos for discussions. This paper was finished while G.H. was visiting the IAS in Princeton and he thanks them

for their hospitality. This work was supported in part by NSF grants PHY-0244764 and PHY99-07949.

\section{References}

[1] J. D. Bekenstein, "Black hole hair: Twenty-five years after," arXiv:gr-qc/9605059.

[2] D. Kastor and J. H. Traschen, "Horizons inside classical lumps," Phys. Rev. D 46 (1992) 5399 arXiv:hep-th/9207070.

[3] R. Emparan and H. S. Reall, "A rotating black ring in five dimensions," Phys. Rev. Lett. 88, 101101 (2002) arXiv:hep-th/0110260. 
[4] H. Elvang, "A charged rotating black ring," Phys. Rev. D 68 (2003) 124016 arXiv:hep-th/0305247; H. Elvang and R. Emparan, "Black rings, supertubes, and a stringy resolution of black hole non-uniqueness," JHEP 0311 (2003) 035 arXiv:hep-th/0310008.

[5] R. Emparan, "Rotating circular strings, and infinite non-uniqueness of black rings," JHEP 0403, 064 (2004) arXiv:hep-th/0402149.

[6] H. Elvang, R. Emparan, D. Mateos and H. S. Reall, "A supersymmetric black ring," Phys. Rev. Lett. 93, 211302 (2004) arXiv:hep-th/0407065.

[7] I. Bena and N. P. Warner, "One ring to rule them all ... and in the darkness bind them?," arXiv:hep-th/0408106.

[8] H. Elvang, R. Emparan, D. Mateos and H. S. Reall, "Supersymmetric black rings and three-charge supertubes," arXiv:hep-th/0408120.

[9] J. P. Gauntlett and J. B. Gutowski, "General concentric black rings," arXiv:hep-th/0408122.

[10] I. Bena, "Splitting hairs of the three charge black hole," arXiv:hep-th/0404073.

[11] J. P. Gauntlett and J. B. Gutowski, "Concentric black rings," arXiv:hep-th/0408010.

[12] D. Garfinkle, "Black string traveling waves," Phys. Rev. D 46 (1992) 4286 arXiv:gr-qc/9209002.

[13] F. Larsen and F. Wilczek, "Classical Hair in String Theory I: General Formulation," Nucl. Phys. B 475 (1996) 627 arXiv:hep-th/9604134.

[14] G. T. Horowitz and D. Marolf, "Counting states of black strings with traveling waves," Phys. Rev. D 55 (1997) 835 arXiv:hep-th/9605224.

[15] G. T. Horowitz and H. Yang, "Black strings and classical hair," Phys. Rev. D 55, 7618 (1997) arXiv:hep-th/9701077.

[16] N. Kaloper, R. C. Myers and H. Roussel, "Wavy strings: Black or bright?," Phys. Rev. D 55 (1997) 7625 arXiv:hep-th/9612248.

[17] G. W. Gibbons, D. Kastor, L. A. J. London, P. K. Townsend and J. H. Traschen, "Supersymmetric selfgravitating solitons," Nucl. Phys. B 416, 850 (1994) arXiv:hep-th/9310118. 
[18] J. P. Gauntlett, J. B. Gutowski, C. M. Hull, S. Pakis and H. S. Reall, "All supersymmetric solutions of minimal supergravity in five dimensions," Class. Quant. Grav. 20 (2003) 4587 arXiv:hep-th/0209114.

[19] D. Marolf, arXiv:hep-th/0006117.

[20] H.S.Reall, "Higher dimensional black holes and supersymmetry," Phys. Rev. D 68 (2003) 024024; erratum ibid 70, 089902 (2004), arXiv:hep-th/0211290.

[21] I. Bena, C. W. Wang and N. P. Warner, "Black rings with varying charge density," arXiv:hep-th/0411072.

[22] M. Cyrier, M. Guica, D. Mateos, A. Strominger, "Microscopic Entropy of the Black Ring", arXiv:hep-th/0411187.

[23] I. Bena and P. Kraus, "Microscopic description of black rings in AdS/CFT," arXiv:hep-th/0408186. 